\title{
L'expression verbale de la douleur chez l'enfant : Comparaison intermodale entre sensation de douleur et manipulation tactile
}

\author{
Margaux Bienvenu Psychologiste $\mathrm{PhD}^{1}$, Denis Jacquet $\mathrm{PhD}^{2}$, Marjolaine Michelutti Psychologiste ${ }^{3}$, Chantal Wood MD ${ }^{1}$
}

M Bienvenu, D Jacquet, M Michelutti, C Wood. L'expression verbale de la douleur chez l'enfant : Comparaison intermodale entre sensation de douleur et manipulation tactile. Pain Res Manage 2011;16(3):187-191.

HISTORIQUE: La présente étude se situe dans le contexte de l'expression verbale de la douleur chez l'enfant. Elle porte plus particulièrement sur la dimension qualitative de la sensation de douleur.

OBJECTIF: Nous cherchons à repérer les particularités de l'expression verbale relativement à l'aspect qualitatif de la douleur.

MÉTHODOLOGIE: La recherche a été menée auprès de 60 patients de quatre à 18 ans ressentant de la douleur, rencontrés dans un hôpital universitaire pédiatrique. Elle confirme en premier lieu l'origine des descripteurs sensoriels de la douleur, qui renvoient aux expériences perceptives passées de l'enfant, non nécessairement liées à la douleur. Ces expériences sont qualifiées de prototypiques, dans la mesure où, bien qu'elles soient liées à des contextes de vie variés, le type de rapport au monde qu'elles provoquent ne varie pas.

RÉSULTATS: Dans ce cadre, le pincement, le tiraillement, le tapement, l'écrasement et l'appuiement, le picotement et le serrement constituent chacune des expériences sensorielles et motrices particulières dont la structure de base ne varie pas d'un contexte à l'autre. En second lieu, les résultats obtenus montrent que dès quatre ans, l'enfant est en mesure de comparer, puis de reconnaître une analogie entre une expérience exclusivement tactile et sa sensation de douleur.

CONCLUSION: Ces résultats mettent en lumière le rôle primordial du raisonnement analogique dans l'expression verbale de la douleur, ce qui amène à affirmer que le niveau de développement cognitif de l'enfant n'est pas une variable a priori déterminante lorsqu'il s'agit de qualifier sa douleur.

\author{
Verbal expression of pain in children: Intermodal \\ comparison between pain sensation and tactile \\ manipulation
}

BACKGROUND: The present study was set in the context of verbal pain expression in children and concerns, more exactly, the qualitative dimension of painful sensations.

OBJECTIVE: To identify the peculiarities of verbal expressions related to the qualitative aspect of pain.

METHODS: Sixty patients presenting with pain at a university pediatric hospital were included in the study. Their ages ranged from four to 18 years. The origin of sensorial pain descriptors was confirmed, reflecting the past perceptive experiences of children that are not necessarily painful. These experiences are characterized as prototypes because, although they are related to various contexts of life, their type of interaction with the world does not vary.

RESULTS: In such a context, pinching, tugging, palpitation, squashing and pressing, tingling and squeezing each convey particular sensorial and motor experiences whose basic structure does not change from one situation to another. The results also showed that from four years of age onward, children are able to compare and recognize an analogy between an exclusively tactile experience and their painful sensation.

CONCLUSION: The results emphasize the central role of analogical reasoning in the verbal expression of pain, showing that the level of cognitive development is not an a priori determinant variable for qualifying pain.

Mots clés : analogie; expériences perceptives; perception douloureuse; perception tactile

T a présente recherche fait suite à une étude princeps intitulée : L L'expression verbale et non verbale de la sensation douloureuse chez l'enfant et chez l'adulte : descripteurs sensoriels de la douleur et pensée analogique » (1). Cette dernière étude a montré que, quel que soit l'âge ou la pathologie, il n'existe pas de différence entre enfants ressentant de la douleur d'âges différents et entre enfants (dès quatre ans) et adultes ressentant de la douleur quant à la nature des descripteurs sensoriels produits pour décrire l'aspect qualitatif de la douleur. Ces descripteurs sensoriels ont pour particularité commune de faire référence aux expériences perceptives passées du patient, non nécessairement liées à la douleur. Certes, la diversité, le nombre et la spontanéité des réponses produites par les patients varient en fonction de l'âge, mais leur nature ne change pas.

Ces résultats nous ont amenés à attribuer à ces descripteurs sensoriels de la douleur le statut d'expériences perceptives prototypiques, qui renvoient aux expériences perceptives passées du patient. Cette hypothèse est attestée par la présente étude menée auprès de 60 enfants de quatre à 18 ans ressentant de la douleur.

\begin{abstract}
PARTIE THÉORIQUE
L'expression verbale de la douleur

Il n'existe pas de traduction objective du vécu de douleur, dans la mesure où il s'agit d'une expérience sensorielle personnelle et singulière et non d'une entité conceptuelle. Lors des entretiens d'évaluation de la douleur, les patients tentent de donner à l'expression de leur douleur une objectivité qui la rend transmissible et compréhensible pour l'autre. Plusieurs types de réponses sont produites par les patients (2) : la douleur est localisée, mesurée, ou les conditions de son apparition sont spécifiées. Certains patients ne semblent pas parvenir à surmonter une certaine pauvreté lexicale du champ de la douleur. Cela va du refus de la formulation précise (« je ne sais pas »), à la simple modélisation de l'expression « avoir mal » (un peu, toujours, moins, etc.) D'autres déploient au contraire une palette variée pour verbaliser leur douleur, en diversifiant leurs choix lexicaux. Le recours à des procédés analogiques (métaphores) permet de surmonter une certaine pauvreté lexicale du champ de la douleur (« ça tape comme un marteau »).
\end{abstract}

${ }^{1}$ Unité d'évaluation et de traitement de la douleur, Centre hospitalier universitaire Robert Debré, Paris; ${ }^{2}$ Laboratoire de psychologie des actions langagières et motrices, PPF Modescos, Maison de la recherche en sciences humaines, Université de Caen Basse-Normandie, Caen; ${ }^{3}$ Hôpital local Maurice Fenaille, Engayresque Séverac-le-Chateau, France

Correspondance: Dr Margaux Bienvenu, Unité d'évaluation et de traitement de la douleur, Centre hospitalier universitaire Robert Debré, Paris 75019, France. Télephone 01-40-03-53-46, courriel margaux.bienvenu@rdb.aphp.fr 
La nature des descripteurs sensoriels produits pour décrire l'aspect qualitatif de la douleur est semblable pour les enfants d'âges différents (dès quatre ans) ressentant de la douleur et entre enfants et adultes ressentant de la douleur (1). Parmi ces descripteurs sensoriels, certains sont récurrents : le pincement, le tiraillement, le tapement, l'écrasement ou l'appuiement, le picotement et le serrement.

\section{Perception et saillance perceptive}

D'après nous, la description que l'enfant fait de sa douleur est directement liée aux éléments saillants qu'il aura perçus dans la sensation de douleur elle-même. Par exemple, l'enfant décrit sa douleur en disant que « ça tape ", car il aura perçu la composante pulsatile de la sensation comme saillante (3). Cela nous amène à étendre la notion de saillance perceptive au domaine de la douleur. La notion de saillance (ou prégnance) est avant tout liée à l'émergence d'une figure sur un fond et elle est issue de l'analyse de la perception visuelle. La perception visuelle fait largement intervenir la notion de saillance : notre attention s'arrête prioritairement sur les éléments saillants qui ressortent de notre environnement visuel, jusqu'à axer les processus cognitifs sur ces seuls éléments. À cet égard, la notion de codes de reconnaissance rejoint celle de saillance. Pour Eco (4), lors du processus même de perception, il apparaît que « nous sélectionnons les aspects fondamentaux du perçu d'après des codes de reconnaissance; ainsi, lorsqu'au jardin zoologique, nous voyons de loin un zèbre, les éléments que nous reconnaissons immédiatement (et que notre mémoire retient) sont les rayures et non pas la silhouette qui ressemble vaguement à celle d'un âne ou d'un mulet (...) Les codes de reconnaissance (comme les codes de perception) concernent les aspects pertinents. » (4)

\section{La catégorisation et l'analogie}

Lorsque l'enfant décrit sa douleur, il effectue une activité de catégorisation. La catégorisation est la conduite adaptative fondamentale par laquelle l'individu " découpe » le réel physique et social. Sa fonction cognitive est la création de catégories (d'objets, d'individus, etc.) Il s'agit d'un processus adaptatif visant à réduire la complexité du monde. Dans certains cas, cette activité est fondée sur un raisonnement de type logique et dans d'autres, sur un raisonnement de type analogique. Les recherches de ces trente dernières années sur la catégorisation $(5,6)$ amènent à une évolution progressive d'une conception rigide vers une conception flexible de la catégorisation.

Les travaux qui convergent vers une approche flexible de la catégorisation tendent à considérer l'analogie comme étant fondamentalement un mécanisme de catégorisation (7). En effet, analogie et catégorisation peuvent être vues comme un continuum de situations dans lesquelles une connaissance, source ou catégorie, est utilisée pour appréhender une situation nouvelle. L'individu recourt à l'analogie lorsqu'il affronte des situations dans lesquelles la cible est peu explicite ou pose problème, comme la douleur. La fonction psychologique de l'analogie est principalement d'appréhender une situation (la cible) en la traitant comme une autre (la source). Par exemple, l'enfant qui ressent de la douleur et compare sa douleur à « de la mousse », rapproche de façon analogique sa sensation de douleur (la cible) à son expérience perceptive de la mousse (la source).

\section{Les domaines fondamentaux d'expérience}

Pour Lakoff et Johnson (8), « la compréhension de certaines émotions, non clairement définies dans notre expérience, est saisie au moyen de concepts que nous comprenons en termes plus clairs ». La douleur entre dans cette perspective, car elle est en partie une émotion et son expression est complexifiée par son statut sensoriel, ce qui explique qu'elle est abordée au moyen d'autres concepts qui apparaissent plus clairs. Dans ce cadre, la compréhension concerne des «domaines fondamentaux d'expérience » et non des concepts isolés. Un domaine fondamental d'expérience est un ensemble structuré à l'intérieur de l'expérience humaine, conceptualisé par une gestalt expérientielle. Le terme gestalt expérientielle renvoie à la modélisation cognitive des domaines fondamentaux d'expérience. Ces gestalts expérientielles caractérisent des touts structurés dans les expériences humaines fréquentes. Elles représentent des organisations cohérentes des expériences humaines en termes de dimensions naturelles (partie, étape, cause, etc.). Elles sont le produit du fonctionnement corporel (appareil perceptuel et moteur, capacités mentales, structure affective, etc.), des interactions avec l'environnement physique (se mouvoir, manipuler des objets, manger, etc.), sociales à l'intérieur d'une culture (institutions sociales, politiques, économiques et religieuses). Ces gestalts expérientielles sont en fait les produits de la nature humaine.

Lorsque l'enfant évoque un domaine fondamental d'expérience, il effectue une activité de catégorisation. Les catégories d'objets évoquées possèdent certaines propriétés : perceptive (directement fondée sur l'appréhension de l'objet par notre appareil sensoriel), motrice (fondée sur la nature de nos interactions motrices avec les objets), fonctionnelle (fondée sur notre conception des fonctions de l'objet), et intentionnelle (fondée sur les utilisations que nous faisons d'un objet dans une situation donnée). Les catégories d'objets peuvent être décrites comme des gestalts expérientielles possédant au moins une de ces dimensions naturelles, dont chacune définit des propriétés d'interaction spécifiques de l'individu avec son environnement. Les propriétés décrites par ces dimensions ne sont pas des propriétés qui appartiennent en propre aux objets. En effet, les dimensions naturelles des catégorisations (perceptives, fonctionnelles, etc.) naissent des interactions de l'individu avec le monde. Ces propriétés d'interaction sont fondées sur l'appareil sensoriel et moteur, sur les conceptions que se font les hommes des fonctions qu'ils assignent aux objets, etc., et elles donnent aux gestalts expérientielles suffisamment de structure interne pour servir à définir d'autres concepts. Leur structure interne permet ainsi d'avoir prise sur les domaines fondamentaux d'expérience qui sont moins concrets ou moins clairement définis, comme, par exemple, la douleur.

Pour résumer, lorsque l'enfant décrit sa douleur, il procède à une activité de catégorisation en se reportant à ses expériences perceptives passées, sur la base d'une analogie perçue entre un type d'expérience perceptive passée et la douleur, et fondée sur la dimension perceptive, motrice, fonctionnelle ou encore intentionnelle de cette expérience perceptive. La catégorisation, dans ce contexte, attribue un rôle fondamental aux interactions perceptives passées de l'enfant, ravivées au cours de l'acte descriptif.

\section{La comparaison intermodale}

Nous pensons que l'enfant est en mesure de reconnaître tactilement une analogie (une équivalence) entre sa douleur et son expérience tactile (haptique). Pour cela, il doit implicitement effectuer une tâche de comparaison intermodale entre la perception de douleur et la perception tactile manuelle. Il y a comparaison intermodale lorsqu'un individu utilise une information obtenue par une modalité pour juger un stimulus équivalent sur une autre modalité. Par exemple, au cours d'une situation de comparaison intermodale, il est demandé au sujet de visualiser un certain objet, puis de retrouver l'objet le plus ressemblant parmi un ensemble d'objets isolés de la composante visuelle, et cela par le fait de la seule composante haptique (tactile). Ce dispositif de comparaison intermodale rejoint les notions de « redondance " ou de «processus vicariants » (9). Dans une situation donnée, chaque individu disposerait de plusieurs possibilités d'intégrer l'information présente en vue de fournir une réponse adaptée, celles-ci pouvant se confirmer mutuellement ou bien se substituer les unes aux autres.

\section{Hypothèse opérationnelle}

\section{EXPÉRIMENTATIONS}

Les patients qui ressentent de la douleur sont en mesure de reconnaître une analogie entre la perception de douleur et la perception tactile, quel que soit leur âge ou leur pathologie.

\section{Population}

Soixante enfants de quatre à 18 ans ressentant de la douleur ont été rencontrés dans un hôpital universitaire pédiatrique. Les patients 
TABLEAU 1

Population du protocole expérimental

\begin{tabular}{lccc} 
& \multicolumn{3}{c}{ Groupes d'âge } \\
\cline { 2 - 4 } & $\mathbf{4}$ à $\mathbf{7}$ ans & $\mathbf{8}$ à $\mathbf{1 2}$ ans & $\mathbf{1 3}$ à $\mathbf{1 8}$ ans \\
\hline Nombre de patients & 7 & 28 & 25 \\
Âge moyen & 5 ans 8 mois & 9 ans 8 mois & 14 ans 4 mois \\
\hline
\end{tabular}

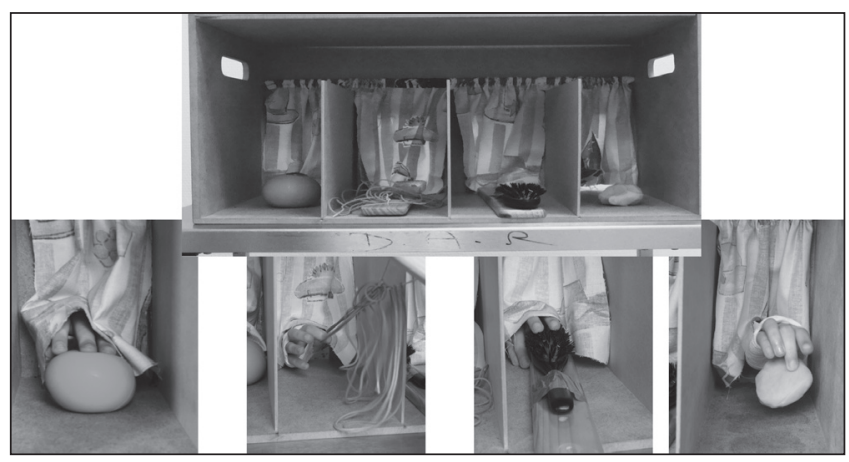

Figure 1 ) Représentations photographiques de la boîte sensorielle

ressentant de la douleur sont répartis ci-dessous en trois groupes d'âges différents (tableau 1).

\begin{abstract}
Méthodologie
Chaque passation a nécessité l'accord des patients ainsi que l'accord signé des parents des patients mineurs. Seule la présence d'une sensation de douleur actuellement présente constitue le critère d'inclusion dans le groupe. Les enfants sont interrogés au cours de leur hospitalisation au sein de différentes services : pédiatrie générale, unité de surveillance continue de chirurgie, gastroentérologie, orthopédie, ORL, chirurgie viscérale et urgences.

Le sexe et la pathologie des patients ne sont pas pris en compte, car ils n'apparaissent pas pertinents au regard de la présente recherche.
\end{abstract}

\section{Matériel}

Un matériel a été spécialement conçu pour mener à bien la recherche. Il s'agit d'une boîte à quatre entrées, qui forment quatre couloirs séparés les uns des autres. La boîte est fermée, afin d'isoler la composante visuelle de ce qui s'y passe à l'intérieur. Un système de planches coulissantes permet cependant à un observateur extérieur de visualiser ou encore d'intervenir à l'intérieur de la boîte, sans que le sujet en ait de perception visuelle (figure 1).

Au fond de chaque couloir se trouvent quatre objets différents de par leurs caractéristiques (tableau 2).

Chaque objet est supposé pouvoir représenter certaines caractéristiques de la sensation de douleur éprouvée par l'enfant, lesquelles ont été relevées au cours de nos précédentes expérimentations et qui font partie des descripteurs sensoriels les plus produits par les patients ressentant de la douleur (enfants et adultes) (1) : le pincement, le tiraillement, le tapement, l'écrasement et l'appuiement, le picotement, le serrement. Par exemple, la brosse semble particulièrement susceptible d'évoquer le picotement.

Autrement dit, les objets contenus dans la boîte ont ainsi été sélectionnés selon le principe que leurs caractéristiques sont susceptibles de produire des sensations de type pincement, tiraillement, tapement, écrasement et appuiement, serrement et picotement. Nous nous attendons cependant à ce que les patients interrogés soulignent des caractéristiques non prévues des objets, mais cela n'a pas vraiment d'importance, dans la mesure où c'est le rapport entre les verbalisations pré- et post-manipulations tactiles qui nous intéresse. De ce fait, nous ne souhaitons pas présenter a priori de correspondances figées entre un type de descripteur sensoriel et un type d'objet.
TABLEAU 2

Caractéristiques des objets de la boîte sensorielle

\begin{tabular}{ll}
\hline Type d'objet & Caractéristiques de l'objet \\
\hline Balle & $\begin{array}{l}\text { Molle, flexible, souple, déformable, retrouve sa forme } \\
\text { initiale après manipulation }\end{array}$ \\
Élastiques & $\begin{array}{c}\text { Multiples, longs, étirables, retrouvent leur forme initiale } \\
\text { après manipulation }\end{array}$ \\
Brosse & $\begin{array}{l}\text { Picots montés sur une structure plate et rigide } \\
\text { Pâte à modeler }\end{array}$ \\
& $\begin{array}{l}\text { Molle, flexible, souple, déformable, ne retrouve pas sa } \\
\text { forme initiale après manipulation }\end{array}$ \\
\hline
\end{tabular}

TABLEAU 3

Questionnaire associé à la passation de la boîte sensorielle

\section{Âge, sexe, pathologie}

DESCRIPTION DE LA SENSATION

Introduction : « Je vais te poser quelques questions pour mieux comprendre à quoi ressemble ta (tes) douleur(s). »

«TA (TES) DOULEUR(S), ÇA FAIT COMMENT ? :...

Maintenant, pour que je puisse mieux comprendre comment ça fait quand tu as mal, tu peux utiliser cette boîte. Tu vois (en montrant) cette boîte, elle a quatre fenêtres et si tu passes ta main dans chaque fenêtre sans voir à l'intérieur, tu peux toucher quelque chose avec ta main.

Dans chaque fenêtre, il y a des choses différentes et tu pourras mettre ta main dedans pour me dire si ce que tu touches peut faire pareil que quand tu as mal. Tu es d'accord?

À chaque fenêtre :

Tu peux maintenant mettre ta main dans la première fenêtre et toucher ce qu'il y a à l'intérieur.

Tu sens ? Tu peux prendre tout le temps dont tu as besoin pour toucher ce qu'il y a à l'intérieur.

Ça fait comment ce que tu touches?

Alors maintenant, tu peux me dire si ce que tu touches peut faire pareil que quand tu as mal ? et pourquoi ? (la question est réitérée à chaque objet) ».

Déroulement de la passation

La passation est standardisée et s'appuie sur un ensemble de questions préétablies (tableau 3 ).

Dans un premier temps, l'enfant est interrogé sur la qualité de sa sensation de douleur, de façon à mettre en évidence par la suite les rapports entre verbalisations pré- et post-manipulations tactiles. Dans un second temps, et seulement si le ou les descripteurs sensoriels produits par l'enfant (ou l'adulte) pour décrire sa douleur sont de type pincement, tiraillement, tapement, écrasement, picotement et serrement, la boîte sensorielle est utilisée. L'enfant est invité à glisser successivement sa main dans chacune des quatre fenêtres, puis à décrire les objets manipulés ( «̧̧a fait comment, ce que tu touches?») avant de les comparer à son éprouvé de douleur («Alors maintenant, tu peux me dire si ce que tu touches peut faire pareil que quand tu as mal ?»). Les réponses des enfants s'expriment en termes de réponses positives ou négatives avec ou sans justification (spontanées ou sollicitées par l'expérimentateur : «Pourquoi ça peut [ne peut pas] faire pareil que quand tu as mal ?»).

\section{RÉSULTATS}

Notre objectif consistait d'abord à juger de l'existence de liens entre la perception de douleur et la perception tactile. Pour cela, nous avons analysé les données en termes de réponses positives et négatives avec justifications. Cela signifie que nous considérons que le patient effectue un lien analogique lorsqu'il reconnaît explicitement que la manipulation tactile peut faire pareil que quand il a mal et lorsqu'il rejette l'existence d'un lien analogique entre les deux types d'expériences et qu'il parvient à justifier ce rejet. Par exemple, l'enfant 


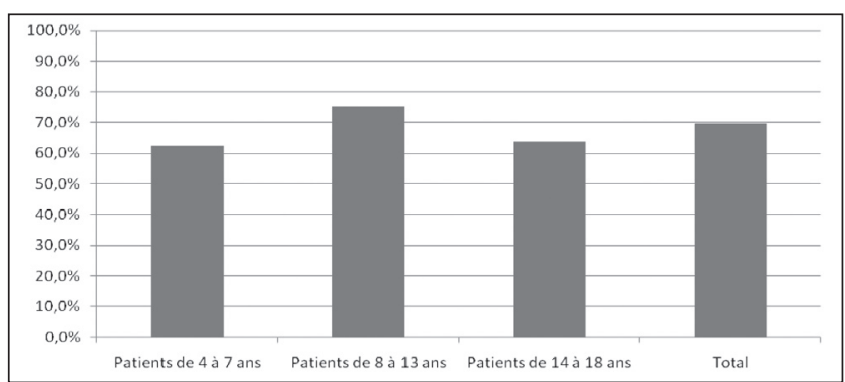

Figure 2 ) Pourcentages de liens analogiques au protocole expérimental $n^{\circ} 2$

qui dit de sa douleur que «ça tire », va juger que la manipulation tactile des élastiques « peut faire pareil que quand il a mal » ou bien que «ça na peut pas faire pareil que quand il a mal, car lui, il ressent que ça pique ». A contrario, nous estimons que les réponses négatives sans justification et les réponses de type «je ne sais pas », sont le reflet d'une absence de lien analogique entre les deux types d'expériences.

Comme prévu, parmi les patients rencontrés qui ressentent de la douleur, un grand nombre font référence aux expériences de pincement, tiraillement, tapement, écrasement, picotement et serrement pour décrire leur douleur $(n=60)$. Ces descripteurs sensoriels font communément référence aux perceptions passées du patient ressentant de la douleur. Seuls les patients ayant produit ce type de réponse sont concernés par la passation de la « boîte sensorielle ».

Nous avons procédé au calcul de pourcentages de liens entre les descripteurs sensoriels produits par les patients et les objets manipulés à l'intérieur de la " boîte sensorielle ». Les calculs ont été effectués pour l'ensemble des patients qui ressentent de la douleur et par groupes d'âge (quatre à sept ans, huit à 12 ans et 13 à 18 ans). Dans un second temps, par le biais d'un test de comparaisons de pourcentages, nous avons procédé à la comparaison des pourcentages de liens pour les trois groupes d'âge.

Il apparaît que 69,7 \% des patients ressentant de la douleur admettent l'existence d'une analogie entre les perceptions douloureuse et tactile : ils sont $62,5 \%$ entre quatre et sept ans, 75,3\% entre huit et 12 ans et $63,8 \%$ entre 13 et 18 ans. Nous n'observons pas de différences significatives de réponses entre les trois classes d'âge, excepté entre les patients de huit à 12 ans et de 13 à 18 ans, pour lesquels nous constatons une très légère différence de réponses, qui s'exprime en terme de liens analogiques plus nombreux chez les patients de huit à 12 ans (figure 2).

\section{EXPOSÉ}

L'objectif du protocole expérimental était de tester les compétences des patients ressentant de la douleur en matière de comparaison intermodale, concernant la perception de douleur et la perception tactile. Dans ce contexte, le patient qui dit de sa douleur que «ça pique », est susceptible d'estimer que la manipulation tactile de la brosse peut faire pareil que sa douleur, bien que la brosse soit une entité dont la nature et la fonction diffèrent de celles de la douleur. Ces résultats nécessitent que l'individu ait, dans un premier temps, effectué un travail cognitif de comparaison intermodale et dans un second temps, qu'il ait repéré un élément équivalent, redondant entre les deux types de modalités. Cet élément équivalent, ou encore analogue, correspond à l'élément saillant issu de la sensation douloureuse. Cet élément saillant est en quelque sorte l'élément fondateur de la comparaison intermodale.

Il ressort de l'analyse des résultats que la majorité des patients parviennent à établir des liens entre les deux types de perceptions. Cela signifie que, quel que soit leur âge ou leur pathologie, la majorité des patients sont en mesure de reconnaître qu'une sensation tactile ne provoquant pas de douleur peut partager une analogie avec une sensation douloureuse. En terme de nombre de réponses, il apparaît qu'il existe une légère différence de résultats entre les patients de huit à
12 ans et ceux de 13 à 18 ans. Cette différence s'exprime en termes de liens analogiques plus nombreux chez les patients de huit à 12 ans.

Nous préférons être critiques quant à la nature même des associations effectuées par les patients ressentant de la douleur entre un type de descripteur sensoriel et un type d'objet. En effet, nous reconnaissons l'existence d'un lien analogique, à la fois lorsque le patient reconnaît explicitement que la manipulation tactile peut faire pareil que quand il a mal, mais aussi lorsqu'il rejette l'existence de ce lien et qu'il parvient à justifier ce rejet. En conséquence, les liens que nous avons mesurés ne permettent pas d'estimer avec exactitude quelles sont les associations « réelles » effectuées par le patient entre un type de descripteur sensoriel et un type d'objet. Cela signifie que rien ne permet d'affirmer avec exactitude que les liens mesurés entre un descripteur sensoriel et un objet reflètent les correspondances effectuées implicitement par les patients. Par exemple, nous considérons qu'il a un lien analogique entre la perception de douleur et la perception tactile, lorsque l'enfant dit de sa douleur que «ça tape » et de la boule « qu'elle ne peut pas faire pareil, parce que c'est tout mou et que son doigt n'est pas tout mou et ne rebondit pas ». Cela ne signifie pas que la boule «tape ", mais plutôt que la boule ne tape pas et que c'est pourquoi elle n'est pas pareille à la douleur. Or, cette réponse fait apparaître conjointement la boule et le tapement dans notre analyse des résultats. De même, le patient qui ressent de la douleur, qui dit de sa douleur que «ça pince » et qui affirme que la manipulation tactile des élastiques peut faire pareil que quand il a mal, peut se reporter en fait implicitement à la sensation de tiraillement qu'il n'aura pas détectée ou signalée explicitement. Un autre cas de figure est celui des réponses multiples. Il s'agit des patients qui produisent plusieurs types de descripteurs sensoriels pour décrire leur douleur et pour lesquels il devient difficile de juger de la place de chacun dans les liens effectués par les patients. C'est le cas, par exemple, pour le patient qui dit de sa douleur que «ça pique et ça pince » et qui estime que la manipulation tactile de la boule " peut faire pareil que quand il a mal ». Il est alors difficile de savoir si le lien effectué par le patient entre la perception de douleur et la perception tactile fait implicitement référence au picotement ou bien au pincement, ou peut-être même à un autre type de descripteur sensoriel non signalé.

Le faible nombre de patients âgés de quatre à sept ans est causé par des facteurs circonstanciels non contrôlables. En effet, peu d'enfants de cet âge correspondant aux critères de passation étaient hospitalisés au moment des expérimentations. La faible quantité de données fragilise les résultats obtenus, mais nous estimons que ces données constituent malgré tout un bon indice des compétences des enfants de cet âge.

Cela nous amène à conclure que notre analyse des résultats valide l'existence de liens analogiques entre la perception de douleur et la perception tactile, mais ne permet pas d'émettre d'affirmations quant à la nature même de ces liens. Ainsi, il convient d'être prudent quant aux associations qui apparaissent comme dominantes, entre un type de descripteur sensoriel et un type d'objet. A posteriori, nous pensons que seule une analyse plus poussée des justifications des patients ressentant de la douleur serait susceptible de lever les ambiguïtés de réponses que nous venons d'évoquer.

Ces résultats confirment le fait qu'il existe des expériences perceptives prototypiques, dont l'individu, quel que soit son âge, a fait l'expérience concrète au cours de ses interactions sensorielles avec son environnement. C'est ainsi que le pincement, le tiraillement, le tapement, l'écrasement et l'appuiement, le picotement et le serrement constituent des entités éprouvées dans divers contextes de vie, mais dont le rapport à l'individu est invariant. Ainsi, l'expérience du tapement peut aussi bien être rattachée au tapement du marteau sur un support qu'au tapement dans les mains. L'information sensorielle vécue dans ces deux contextes a des points de convergence qui créent la perception invariante. Le tapement devient alors un domaine fondamental d'expérience.

Il existe des domaines fondamentaux d'expérience en jeu dans l'expression verbale de la douleur, comme le chaud, le froid, les décharges électriques, les fourmillements, etc., que nous n'avons pas pris en 
compte au cours de l'expérimentation, pour des raisons d'ordre méthodologique. Parmi ces descripteurs sensoriels, certains, comme les décharges électriques ou les fourmillements, semblent être régis par un fonctionnement différent de celui des autres. En effet, nous ne pensons pas que l'individu qui compare sa douleur à une décharge électrique se reporte nécessairement à un vécu réel de décharge électrique. De même, l'expérience de fourmillement ne renvoie à aucun vécu explicite. Nous pensons que pour ces types de descripteurs sensoriels, l'individu procède par glissement analogique entre une sensation source initiale et une sensation source secondaire. Par exemple, l'enfant qui compare sa douleur à un fourmillement peut se référer implicitement à la perception de la mousse et la reporter ensuite au terme de fourmillement, dont la signification est culturellement partagée. Pour le terme de décharge électrique, l'individu peut, par exemple, faire référence à la perception visuelle de la foudre, en sélectionnant son aspect fulgurant, puis reporter cette perception au terme de décharge électrique.

Dans tous les cas, ce qui va guider le patient dans son raisonnement analogique est la perception d'un élément saillant dans la sensation douloureuse qui en constitue en quelque sorte la matrice. En d'autres termes, ce sont les traits saillants partagés entre la sensation douloureuse et les expériences perceptives passées du patient qui vont guider la production verbale des descripteurs sensoriels de la douleur, de même que la comparaison intermodale entre sensation douloureuse et manipulation tactile.

\section{CONCLUSION}

À partir de quatre ans, l'enfant est en mesure de décrire sa douleur par le biais de descripteurs sensoriels, qui sont de surcroit de même type que ceux produits par les enfants plus âgés ou les adultes.
L'ensemble de ces descripteurs sensoriels a pour particularité de renvoyer aux expériences perceptives passées de l'enfant, non nécessairement liées à la douleur et auxquelles nous attribuons le statut d'expériences perceptives prototypiques. Les expérimentations menées auprès des 60 patients interrogés ressentant de la douleur confirment cette hypothèse. En effet, dès quatre ans, l'enfant est en mesure de reconnaître une analogie entre une expérience exclusivement tactile et sa sensation de douleur. Cela nous amène à prôner l'efficience précoce du système de comparaison intermodale fondée sur un transfert par analogie liant perception douloureuse et perception tactile.

\section{RÉFÉRENCES}

1. Bienvenu M, Jacquet D, Wood C. (soumis). Expression verbale et non verbale de la sensation douloureuse chez l'enfant et chez l'adulte : descripteurs sensoriels de la douleur et pensée analogique. Les Archives de psychologie.

2. Maury-Rouan C, Priego-Valverde B. La mise en mots de la douleur. Cognition, langage et développement. Hayen : Mardaga, 2003, p. $159-64$.

3. Landragin F. Saillance physique et saillance cognitive. Corela : 2004, vol. 2.

4. Eco U. La structure absente. Paris : Mercure de France, 1972.

5. Roth EM, Shoben EJ. The effect of context on the structure of categories. Cognitive Psychology, 1983, n¹5, p. 346-78.

6. Richard JF. Les activités mentales. Comprendre, raisonner, trouver des solutions. Paris : Armand Colin, 1995.

7. Sander E. L'analogie, du Naîf au Créatif : analogie et catégorisation. Paris : L'Harmattan, 2000.

8. Lakoff G, Johnson M. Les métaphores dans la vie quotidienne. Paris : Les éditions de Minuit, 1980.

9. Reuchlin M. La psychologie différentielle. Paris : PUF, 1993. 


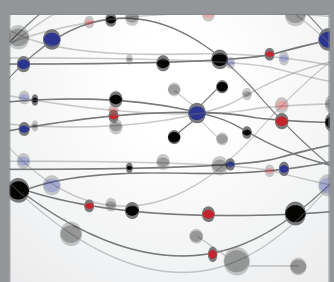

The Scientific World Journal
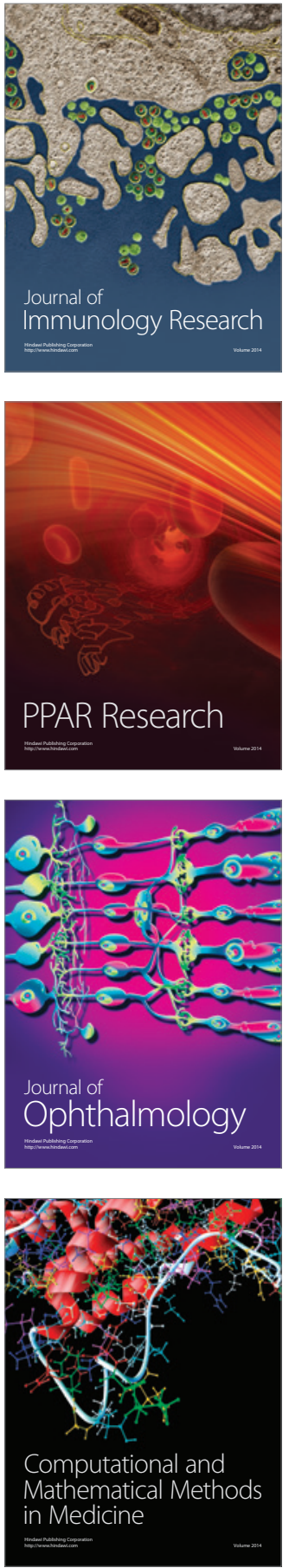

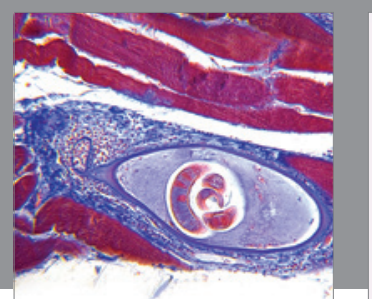

Gastroenterology Research and Practice

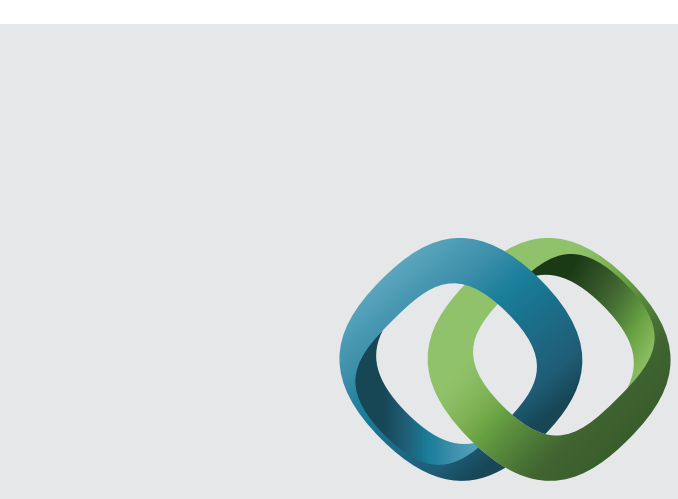

\section{Hindawi}

Submit your manuscripts at

http://www.hindawi.com
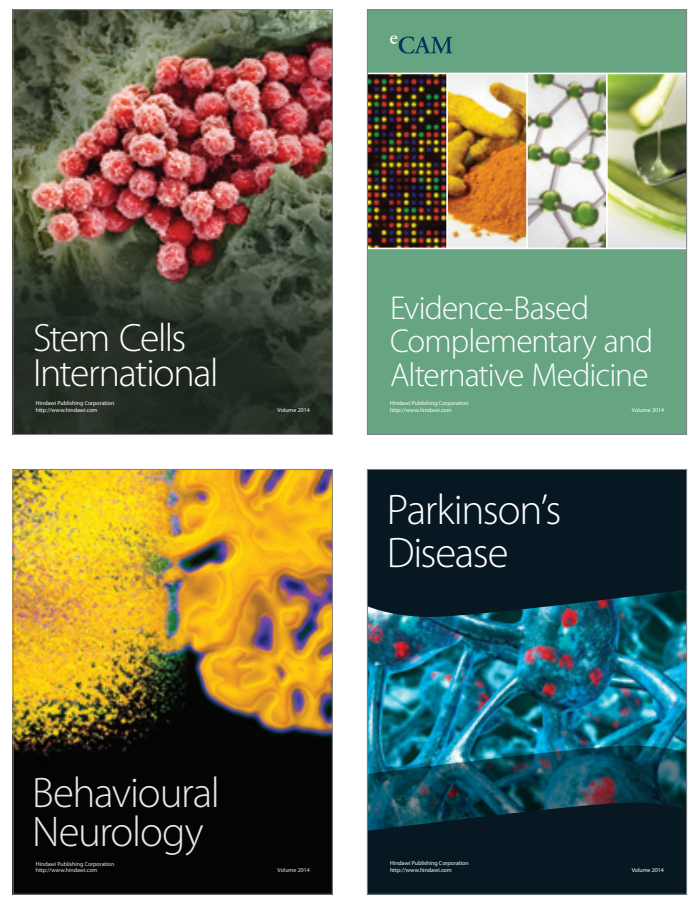
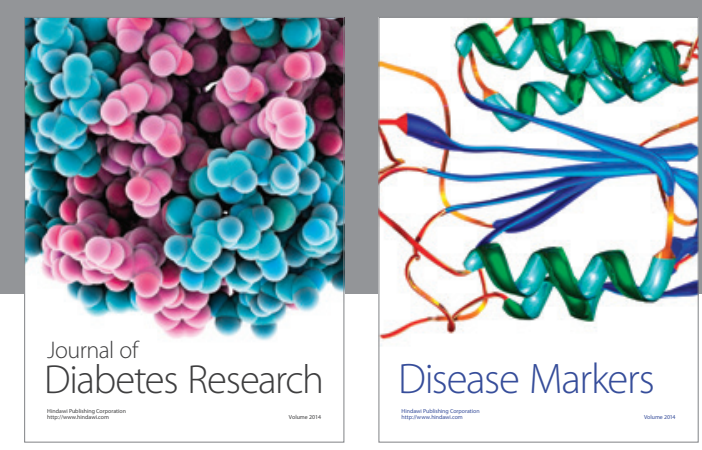

Disease Markers
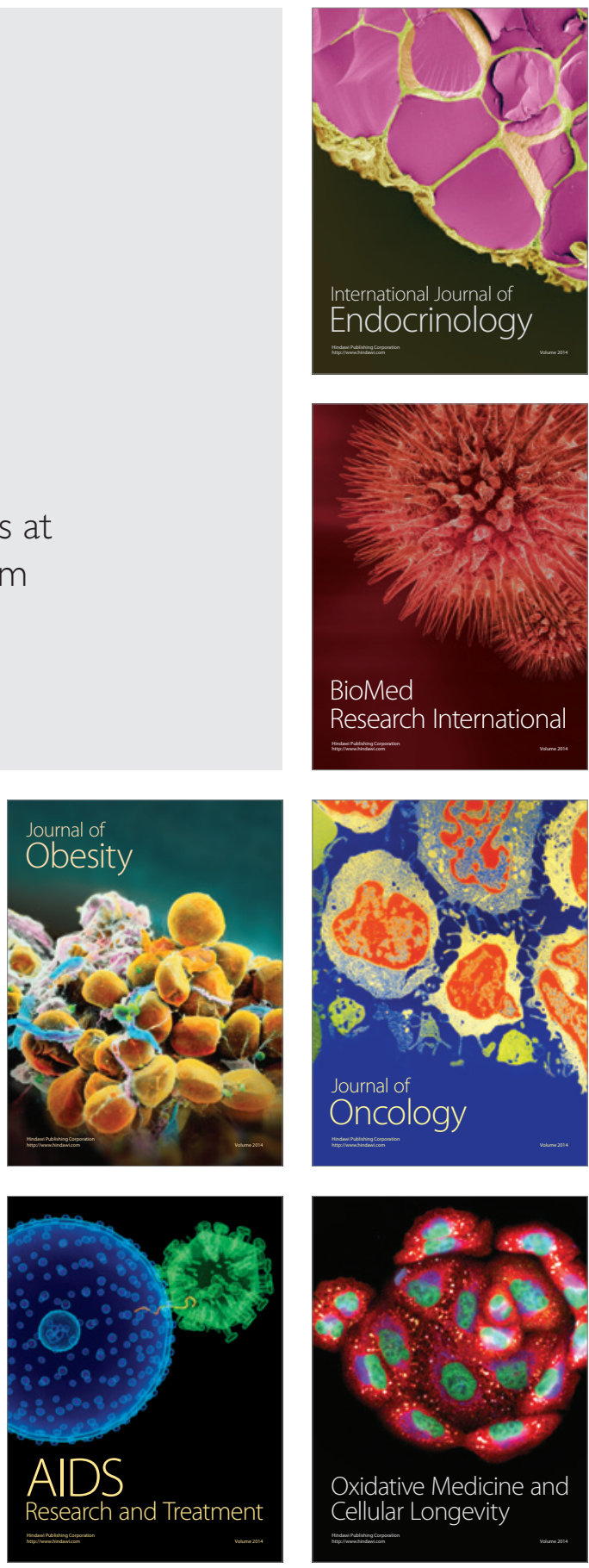